\title{
The influence of knee joint geometry and alignment on the tibiofemoral load distribution: A computational study
}

\section{Sam Van Rossom ${ }^{\mathrm{a}, *}$, Mariska Wesseling a , Colin R. Smith ${ }^{\mathrm{b}, \mathrm{c}}$, Darryl G. Thelen ${ }^{\mathrm{b}, \mathrm{d}, \mathrm{e}}$, Benedicte Vanwanseele ${ }^{\mathrm{a}}$, Van Assche Dieter ${ }^{\mathrm{f}}$, Ilse Jonkers ${ }^{\mathrm{a}}$}

${ }^{a}$ Human Movement Biomechanics Research Group, Department of Kinesiology, KU Leuven, Leuven, Belgium

${ }^{\mathrm{b}}$ Institute for Biomechanics, ETH Zürich, Zürich, Switzerland

c Department of Mechanical Engineering, University of Wisconsin-Madison, Madison, United States

${ }^{d}$ Department of Biomedical Engineering, University of Wisconsin-Madison, Madison, United States

e Department of Orthopedics and Rehabilitation, University of Wisconsin-Madison, Madison, United States

${ }^{\mathrm{f}}$ Musculoskeletal Rehabilitation Research Group, Department of Rehabilitation Sciences, KU Leuven, Leuven, Belgium

\section{A R T I C L E I N F O}

\section{Article history:}

Received 4 July 2018

Received in revised form 14 November 2018

Accepted 1 June 2019

Available online $\mathrm{xxxx}$

\section{Keywords:}

Contact forces

Malalignment

Tibial slope

Ligament strain

Osteoarthritis

\begin{abstract}
A B S T R A C T
Background: Deviations in knee joint geometry and alignment were previously related to an increased risk for knee OA. These were hypothesized to influence the load distribution over the articular cartilage. Therefore, this study evaluated the effect of altered knee joint geometry and alignment in the coronal and transverse plane on the medial-lateral load distribution and ligament strain using a musculoskeletal modeling approach.

Methods: Joint kinematics during gait were measured in 15 healthy adults. Using different musculoskeletal models with altered geometry of the tibia plateau or knee joint malalignment in the coronal and transverse plane, the resulting muscle, ligament and contact forces were calculated. Next, the distribution of the load over the medial and lateral condyle was analyzed and compared to the reference loading distribution, with neutral geometry and alignment, using repeated-measures ANOVA and individual t-tests, with a Bonferroni-corrected alpha level.

Results: Coronal plane malalignment significantly affected the load distribution. Small changes in coronal tibial slope had less pronounced effects on the load distribution, but increased ligament strains. Transverse plane malalignment only minimally affected the load distribution.

Conclusion: Coronal plane knee malalignment affected knee loading, with increased varus alignment resulting in increased medial loading. This confirms a causal relation between coronal malalignment and increased medial compartment loading and suggests a potential role of aberrant coronal plane alignment on OA initiation. Altered coronal tibial slope induced increased ligament strains, potentially contributing to a cascade of knee laxity and subsequently more extreme knee malalignment.
\end{abstract}

(c) 2019 Elsevier B.V. All rights reserved.

\section{Introduction}

Multiple factors contribute to the initiation of knee osteoarthritis (OA) and ultimately articular cartilage breakdown [1]. Population based studies identified altered knee joint geometry and joint alignment in the coronal and transverse plane as risk factors

\footnotetext{
* Corresponding author at: Tervuursevest 101, box 1501, 3001 Leuven, Belgium.

E-mail addresses: sam.vanrossom@kuleuven.be, (S. Van Rossom), mariksa.wesseling@kuleuven.be, (M. Wesseling), crsmith25@wisc.edu, (C.R. Smith), dgthelen@wisc.edu, (D.G. Thelen), benedicte.vanwanseele@kuleuven.be, (B. Vanwanseele), dieter.vanassche@uzleuven.be, (V.A. Dieter), ilse.jonkers@kuleuven.be. (I. Jonkers).
} 
for knee OA development [2-4]. Risk factors for knee OA (e.g. geometry, alignment and knee laxity) are hypothesized to affect the knee load distribution, therefore increasing the stress on the different knee joint structures, resulting in cartilage degeneration [5].

Altered coronal plane joint geometry, more specifically increased elevation of the lateral tibial plateau and an increased coronal tibial slope, was previously related to an increased risk for knee OA initiation by affecting joint loading [6,7]. Apart from its role in altering the knee load distribution and therefore increased risk for knee OA initiation, altered joint geometry may also induce malalignment of the knee joint in a later stage of the OA process itself [7].

Deviations in coronal plane joint alignment, especially varus and valgus malalignment, were previously related to an increased risk for bone marrow lesions and cartilage loss in the medial and lateral condyle, respectively [8-10]. Using the knee adduction moment (KAM) as an indirect measure of medial condyle loading, the degree of varus alignment was found to be directly proportional to KAM [11-13]. This suggests that varus malalignment increases loading on the medial condyle and could therefore contribute to OA initiation and progression [3,14-18]. Furthermore, OA patients with increased internal tibia rotation, an indication of transverse plane malalignment, were found to walk with increased KAM, suggesting increased loading and compression of the medial condyle as a potential contributor to knee OA [19].

Besides their effect on the knee joint loading, altered joint geometry and alignment may influence the stability provided by the ligaments. Given the relation between knee instability and OA, ligamentous laxity was previously suggested to contribute to the initiation and progression of knee OA [20-22]. Ligamentous laxity and the consequent knee instability could cause a shift in contact locations towards infrequently loaded regions. As in these regions the cartilage is less suited for loading, degenerative changes in the cartilage could be induced [5,23]. However, the potential role of altered joint geometry and malalignment in aberrant ligament lengthening behavior and therefore knee instability is currently unexplored.

Despite their clinical significance, the effect of alterations in joint geometry and alignment on knee cartilage loading distribution and ligament strains cannot be investigated in-vivo. Therefore, the role of altered loading in presence of aberrant joint geometry or alignment on the pathomechanics of OA cannot be confirmed. In-vitro, it was found that varus alignment resulted in increased medial condyle pressure under static loading [24,25]. To corroborate these in-vitro findings, musculoskeletal modeling can be used to assess and quantify the isolated effect of alignment or geometry on the contact force distribution over the articular cartilage. Since other influencing factors, such as ligament properties, height, weight can be kept constant [26].

Therefore, the purpose of this study was to investigate systematically the effect of alterations in knee joint geometry and malalignment in the coronal and transverse plane on cartilage loading distribution as well as the ligament strains using a musculoskeletal modeling approach.

\section{Methods}

\subsection{Experimental procedure}

Barefoot walking at self-selected speed $(1.39 \pm 0.12 \mathrm{~m} / \mathrm{s})$ was measured in fifteen healthy adults (eight males, seven females, mean age $=30.73 \pm 5.84$ years, mean weight $=70.49 \pm 7.24$ and mean height $=177.47 \pm 6.61$ ). A 10-camera Vicon system was used to capture three-dimensional marker trajectories (100 Hz, Vicon, Oxford Metrics, Oxford, UK). Markers were positioned according to an extended full-body Plug-in-Gait marker set (Supplementary material A). Simultaneously, ground reaction forces were measured, using two ground-embedded force plates (1000 Hz, AMTI, Watertown, USA). Three trials per subject were retained for further processing. The university hospital ethics committee approved all procedures (s56093) and written informed consent was obtained.

\subsection{Musculoskeletal modeling}

A customized knee joint with six degrees of freedom (DoF) in the patellofemoral and tibiofemoral joint was implemented in a generic lower extremity model [27]. Each leg of the musculoskeletal model contains 44 musculotendon actuators and 14 bundles of non-linear springs, representing the major knee ligaments and posterior capsule. Cartilage contact pressure was calculated based on the penetration depth of the overlapping cartilage surface meshes, using a non-linear elastic foundation formulation [28]. The cartilage was assumed to be uniformly distributed with a combined thickness of four and seven millimeters for the tibiofemoral and patellofemoral joint, respectively [29,30]. The elastic modulus of the cartilage was defined as $10 \mathrm{MPa}$ and Poisson's ratio as 0.45 [31,32]. This model was implemented in SIMM with the Dynamics Pipeline (Musculographics Inc., Santa Rosa, CA) and SD/Fast (Parametric Technology Corp., Needham, MA) was used to generate the multibody equations of motion.

To generate the reference simulations, the generic model was scaled based on the marker locations in a static calibration trial and the subject's mass. Next, an inverse kinematics procedure was used to calculate the joint kinematics based on the measured 3D marker trajectories. Subsequently, the muscle activations required to reproduce the measured primary hip, knee and ankle accelerations were estimated using the concurrent optimization of muscle activations and kinematics algorithm, in which the weighted sum of squared muscle activations and contact energy were minimized [28]. The secondary tibiofemoral and all patellofemoral DoF evolved as a function of muscle, ligament and contact forces, since only the knee flexion angle was prescribed during the optimization $[27,28]$.

To generate the simulations with varied joint alignment and geometry, the scaled musculoskeletal model was modified as described in Figure 1. The effect of variation in joint geometry was investigated by only altering the orientation of the tibia plateau in the coronal plane, while keeping the knee alignment unchanged. This mimicked a more elevated medial or lateral tibia plateau, 


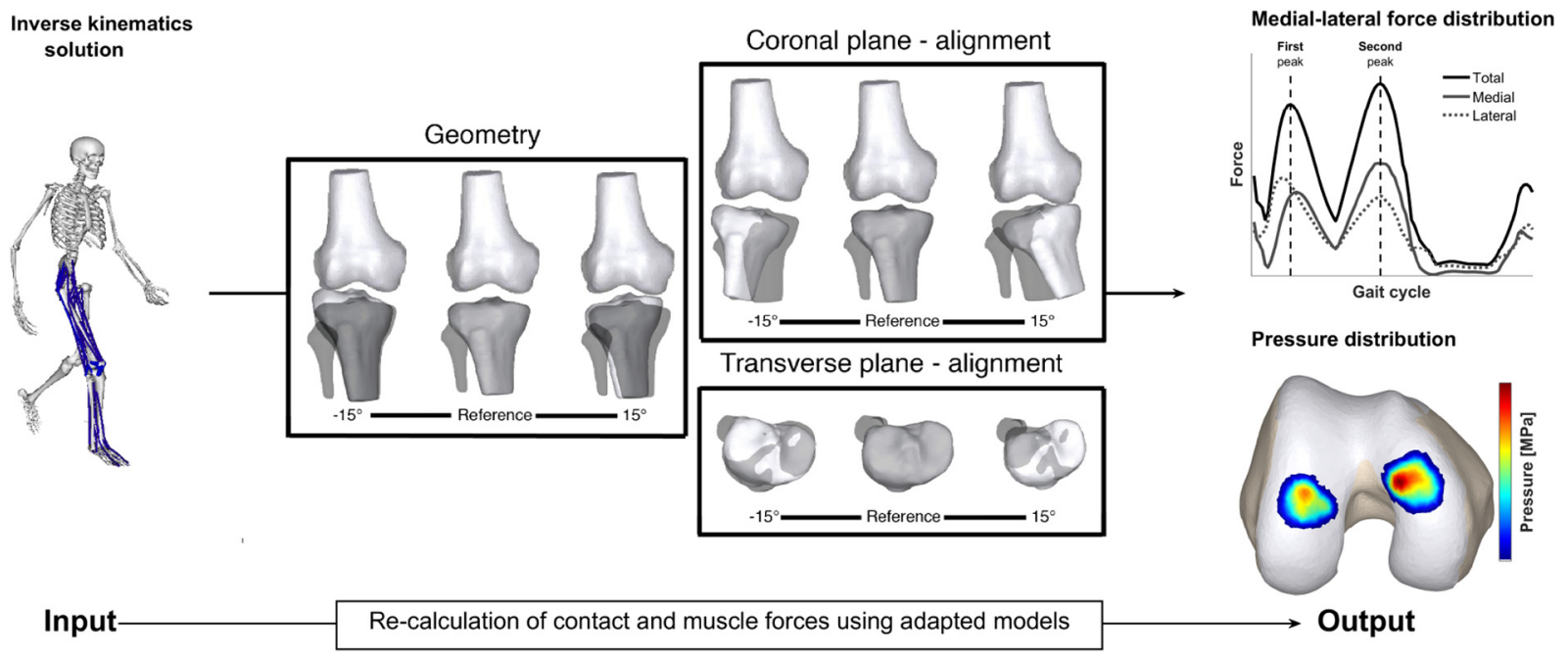

Figure 1. Overview of the methodology. A scaled musculoskeletal model was used to calculate the kinematics during walking. Next, the muscle forces and contact forces were calculated with models in which the alignment of the knee or the position of the tibia plateau was systematically changed from $1^{\circ}$ to $15^{\circ}$ from its reference position in the coronal and transverse plane in steps of $2^{\circ}$. The position of the tibia plateau was changed to simulate a deviating joint geometry. Gray bone indicates the reference position. Subsequently, the effect on the contact force and pressure distribution was analyzed and compared with the reference loading pattern obtained with the original model (gray bones). Note: when changing geometry alignment of the knee was not changed and the femur bone remained unaffected in all models.

representative of altered coronal tibial slope (coronal plane). The effect of malalignments in the coronal and transverse plane was investigated by altering the relative orientation of the tibia relative to the femur in the coronal and transverse plane. This resulted in a more varus or valgus aligned knee (coronal plane) or a more internally or externally rotated knee (transverse plane). Ligament resting length was scaled to compensate for the altered ligament length in the different models due to the altered alignment. For both joint geometry and alignment in the coronal and transverse plane, each parameter was systematically varied from $\pm 1^{\circ}$ to $\pm 15^{\circ}$ from its neutral position in steps of two degrees [3,26]. This resulted in a dataset containing three simulations for each type of modification for each subject. In total, 2295 simulations were generated: 51 simulations for 15 subjects for each type of modification).

After these modifications to the musculoskeletal models, simulations with the reference input kinematics and ground reaction forces (GRF) were generated using the similar modeling workflow as described above (Figure 1). When simulating malalignment, the location of the foot with respect to the measured GRF application point is changed. To ensure that for these simulations the application of the ground reaction force to the foot was identical to the reference simulation, the center of pressure (of the ground reaction force) was expressed in the local reference frame of the foot. We assumed that compensations in the trunk kinematics would account for any dynamic inconsistency [33].

\subsection{Data analysis}

The two peaks in the resultant tibiofemoral contact force were identified and the coinciding knee adduction moment (KAM), contact force magnitude and mean pressure were analyzed. All variables, except KAM were analyzed for the medial and lateral condyle separately. KAM was scaled to body mass, contact forces were scaled to bodyweight (BW) and contact pressures were scaled to bodyweight and knee dimensions $\left(B W * A^{2}\right)$. Furthermore, peak ligament strains were determined for the anterior and posterior cruciate ligament (ACL and PCL), as well as the medial and lateral collateral ligament (MCL and LCL). Ligament strain was calculated by:

$$
\epsilon=\frac{l-l_{0}}{l_{0}}
$$

with $l_{0}$ being the ligament length in the reference position and $l$ being the ligament length.

A repeated-measures ANOVA was used to check for significant differences in contact force, contact pressure and ligament strain between the different geometries or alignments separately. When a significant main effect for geometry or alignment was found, all imposed geometries or alignments were individually compared to the reference simulation using a paired t-test. Significance level was set at $\alpha=0.05$ and a Bonferroni correction was applied to correct for the multiple testing ( $\alpha_{B C}=$ 0.003125). All statistical tests were conducted in MATLAB (MATLAB 2016b, The Math Works, Inc., Natick, Massachusetts, USA). 

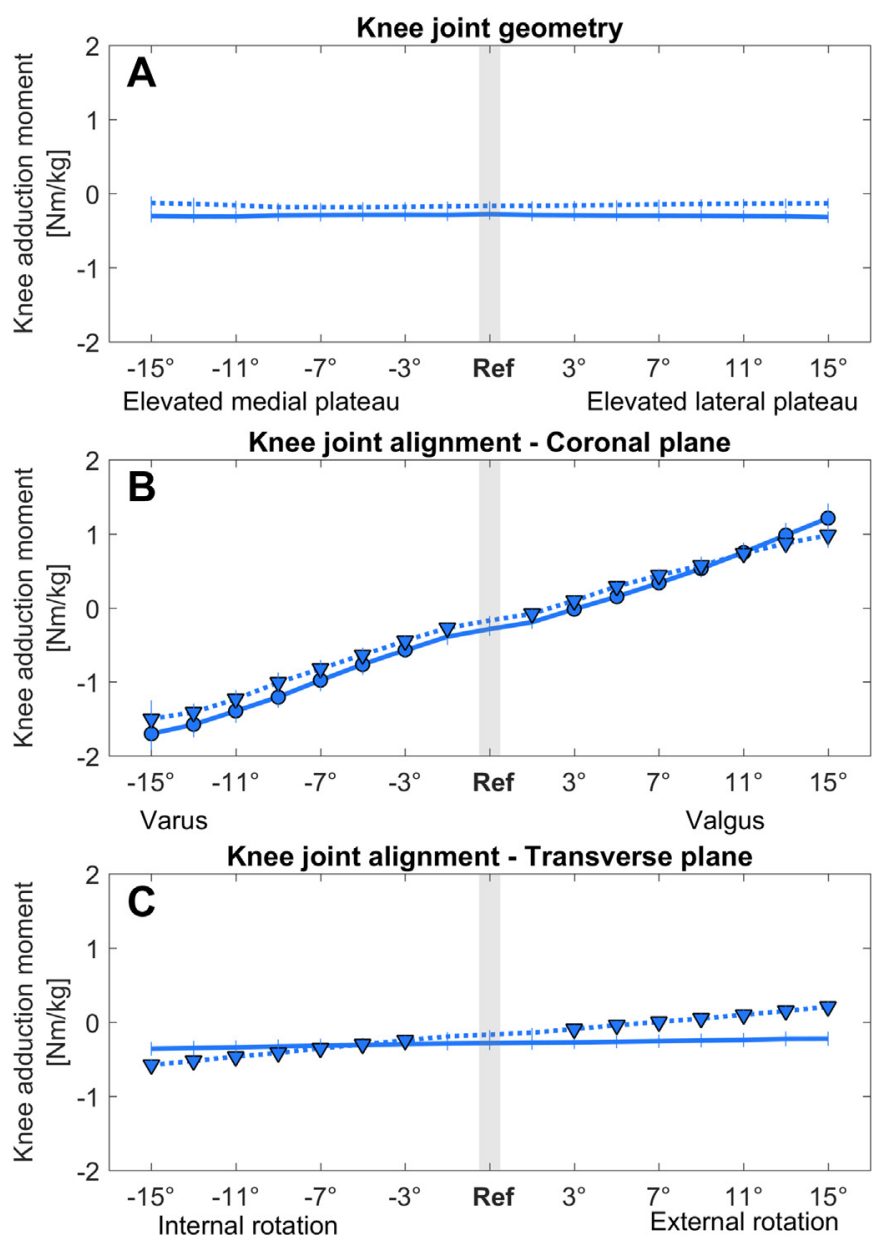

-KAM - First Peak $\cdots$ KAM - Second Peak

Figure 2. Effect on the external knee adduction moment. The effect of an altered joint geometry in A) the coronal plane, C) the transverse plane and an altered joint alignment in B) the coronal plane and D) the transverse plane on the external knee adduction moment at the first peak (FP, solid line) and at the second peak (SP, dashed line). A significant difference compared to the knee adduction moment during the reference simulation (gray bar) is indicated by a solid dot (first peak) and a solid triangle (second peak). ( $\left.\alpha_{B C}=0.0031\right)$.

\section{Results}

\subsection{Knee geometry}

\subsubsection{Coronal plane}

KAM was not significantly affected by the coronal plane geometry of the tibia plateau (Figure 2A, Table 1). However, mediallateral force distribution was significantly altered when the medial or lateral tibial plateau was elevated more than five degrees and seven degrees, respectively (Figure 3A, Table 1). First and second peak medial contact force were significantly increased when the medial plateau was elevated beyond seven degrees. The first and second peak lateral contact force were significantly decreased when the medial plateau was elevated beyond $11^{\circ}$ and five degrees. In contrast, first and second peak lateral contact force were significantly increased when the lateral plateau was elevated beyond nine degrees and seven degrees, respectively. Second peak medial contact force was significantly decreased when the lateral plateau was elevated beyond $13^{\circ}$ (Figure $3 \mathrm{~A}$ ).

Medial mean pressure was significantly increased when the medial plateau was elevated beyond nine degrees and $11^{\circ}$ at the first and second peak, respectively, whereas lateral mean pressure at the second peak was significantly decreased when the medial plateau was elevated beyond seven degrees (Figure 4A). Lateral mean pressure was significantly increased when the lateral plateau was elevated beyond $15^{\circ}$ and nine degrees at the first and second peak, respectively (Figure 4A, Table 1). Results for the effect on the total knee contact force are reported in supplementary material B.

ACL peak strain was significantly increased when the lateral plateau was elevated beyond $13^{\circ}$, whereas PCL peak strain was significantly increased when the medial plateau was elevated beyond $15^{\circ}$ (Figure 5A, Table 1). MCL and LCL peak strain were 
Table 1

Altered geometry and malalignment conditions in the coronal plane resulting in significantly altered loading conditions.

\begin{tabular}{|c|c|c|c|c|c|c|}
\hline \multirow[t]{2}{*}{ Coronal plane - geometry } & & \multirow{2}{*}{$\begin{array}{l}\text { Extreme } \\
\text { Elevated medial } \\
\text { plateau }\end{array}$} & \multirow[t]{2}{*}{ Significant from } & \multirow[t]{2}{*}{ Reference value } & \multirow[t]{2}{*}{ Significant from } & \multirow{2}{*}{$\begin{array}{l}\text { Extreme } \\
\begin{array}{l}\text { Elevated lateral } \\
\text { plateau }\end{array}\end{array}$} \\
\hline & & & & & & \\
\hline & First Peak & - & - & $-0.28 \pm 0.08$ & - & - \\
\hline \multirow[t]{2}{*}{ Knee adduction moment (Nm/kg) } & Second Peak & - & - & $-0.17 \pm 0.06$ & - & - \\
\hline & First Peak & $3.76 \pm 0.22$ & $2.23 \pm 0.2\left(7^{\circ}\right)$ & $1.83 \pm 0.23$ & - & - \\
\hline \multirow{2}{*}{ Medial contact force (BW) } & Second Peak & $3.57 \pm 0.39$ & $2.5 \pm 0.41\left(7^{\circ}\right)$ & $1.89 \pm 0.32$ & $1.53 \pm 0.2\left(13^{\circ}\right)$ & $1.46 \pm 0.18$ \\
\hline & First Peak & $0.76 \pm 0.31$ & $0.95 \pm 0.33\left(11^{\circ}\right)$ & $1.33 \pm 0.31$ & $1.81 \pm 0.28\left(9^{\circ}\right)$ & $2.31 \pm 0.32$ \\
\hline \multirow[t]{2}{*}{ Lateral contact force (BW) } & Second Peak & $0.08 \pm 0.1$ & $0.7 \pm 0.2\left(5^{\circ}\right)$ & $0.98 \pm 0.23$ & $1.4 \pm 0.28\left(7^{\circ}\right)$ & $1.92 \pm 0.3$ \\
\hline & First Peak & $0.013 \pm 0.002$ & $0.01 \pm 0.001\left(9^{\circ}\right)$ & $0.008 \pm 0.001$ & - & - \\
\hline \multirow[t]{2}{*}{ Medial contact pressure $\left(B W * \mathrm{~A}^{2}\right)$} & Second Peak & $0.012 \pm 0.002$ & $0.01 \pm 0.001\left(11^{\circ}\right)$ & $0.008 \pm 0.001$ & - & - \\
\hline & First Peak & - & - & $0.008 \pm 0.002$ & $0.011 \pm 0.002\left(15^{\circ}\right)$ & $0.011 \pm 0.002$ \\
\hline Lateral contact pressure $\left(\mathrm{BW} * \mathrm{~A}^{2}\right)$ & Second Peak & $0.001 \pm 0.001$ & $0.004 \pm 0.001\left(7^{\circ}\right)$ & $0.006 \pm 0.001$ & $0.007 \pm 0.001\left(9^{\circ}\right)$ & $0.008 \pm 0.001$ \\
\hline ACL strain $(\%)$ & Peak strain & - & - & $5.7 \pm 1.51$ & $7.82 \pm 1.28\left(13^{\circ}\right)$ & $8.49 \pm 1.33$ \\
\hline PCL strain (\%) & Peak strain & $0.64 \pm 1.24$ & $0.64 \pm 1.24\left(15^{\circ}\right)$ & $-2.41 \pm 2.49$ & - & - \\
\hline MCL strain (\%) & Peak strain & $18.55 \pm 1.1$ & $5.46 \pm 0.69\left(3^{\circ}\right)$ & $2.43 \pm 1.18$ & $-0.19 \pm 1.52\left(3^{\circ}\right)$ & $-5.26 \pm 2.09$ \\
\hline LCL strain $(\%)$ & Peak strain & $3.06 \pm 2$ & $1.79 \pm 1.16\left(3^{\circ}\right)$ & $4.92 \pm 0.96$ & $7.8 \pm 0.79\left(3^{\circ}\right)$ & $19.72 \pm 0.66$ \\
\hline \multirow[t]{2}{*}{ Coronal plane - alignment } & & Extreme & Significant from & Reference value & Significant from & Extreme \\
\hline & & Varus & & & & Valgus \\
\hline \multirow{3}{*}{ Knee adduction moment (Nm/kg) } & First Peak & $-1.7 \pm 0.22$ & $-0.57 \pm 0.11\left(3^{\circ}\right)$ & $-0.28 \pm 0.08$ & $-0.01 \pm 0.08\left(3^{\circ}\right)$ & $1.21 \pm 0.2$ \\
\hline & Second Peak & $-1.5 \pm 0.25$ & $-0.27 \pm 0.07\left(1^{\circ}\right)$ & $-0.17 \pm 0.06$ & $-0.08 \pm 0.06\left(1^{\circ}\right)$ & $0.98 \pm 0.17$ \\
\hline & First Peak & $5.71 \pm 0.44$ & $2.44 \pm 0.27\left(3^{\circ}\right)$ & $1.83 \pm 0.23$ & $1.25 \pm 0.23\left(3^{\circ}\right)$ & $0 \pm 0$ \\
\hline \multirow[t]{2}{*}{ Medial contact force (BW) } & Second Peak & $8.24 \pm 2.11$ & $2.57 \pm 0.3\left(3^{\circ}\right)$ & $1.89 \pm 0.32$ & $1.53 \pm 0.29\left(3^{\circ}\right)$ & - \\
\hline & First Peak & $0 \pm 0$ & $0.66 \pm 0.27\left(3^{\circ}\right)$ & $1.33 \pm 0.31$ & $2.06 \pm 0.37\left(3^{\circ}\right)$ & $6.1 \pm 0.97$ \\
\hline \multirow[t]{2}{*}{ Lateral contact force (BW) } & Second Peak & $0 \pm 0$ & $0.48 \pm 0.17\left(3^{\circ}\right)$ & $0.98 \pm 0.23$ & $1.62 \pm 0.23\left(3^{\circ}\right)$ & $5.41 \pm 0.49$ \\
\hline & First Peak & $0.016 \pm 0.002$ & $0.01 \pm 0.001\left(3^{\circ}\right)$ & $0.008 \pm 0.001$ & $0.007 \pm 0.001\left(3^{\circ}\right)$ & $0 \pm 0$ \\
\hline \multirow[t]{2}{*}{ Medial contact pressure $\left(B W * A^{2}\right)$} & Second Peak & $0.023 \pm 0.005$ & $0.01 \pm 0.001\left(3^{\circ}\right)$ & $0.008 \pm 0.001$ & $0.007 \pm 0.001\left(3^{\circ}\right)$ & - \\
\hline & First Peak & $0 \pm 0$ & $0.006 \pm 0.002\left(3^{\circ}\right)$ & $0.008 \pm 0.002$ & $0.013 \pm 0.003\left(5^{\circ}\right)$ & $0.022 \pm 0.003$ \\
\hline Lateral contact pressure $\left(\mathrm{BW} * \mathrm{~A}^{2}\right)$ & Second Peak & $0 \pm 0$ & $0.004 \pm 0.001\left(3^{\circ}\right)$ & $0.006 \pm 0.001$ & $0.008 \pm 0.001\left(3^{\circ}\right)$ & $0.019 \pm 0.004$ \\
\hline ACL strain (\%) & Peak strain & $18.75 \pm 2.17$ & $8.61 \pm 1.28\left(9^{\circ}\right)$ & $5.7 \pm 1.51$ & $8.64 \pm 1.94\left(5^{\circ}\right)$ & $22.33 \pm 3.73$ \\
\hline PCL strain (\%) & Peak strain & $10.97 \pm 1.52$ & $-4.19 \pm 1.11\left(3^{\circ}\right)$ & $-2.41 \pm 2.49$ & $1.7 \pm 1.57\left(7^{\circ}\right)$ & $5.29 \pm 0.56$ \\
\hline MCL strain (\%) & Peak strain & $22.56 \pm 1.99$ & $3.78 \pm 1.13\left(1^{\circ}\right)$ & $2.43 \pm 1.18$ & $0.66 \pm 1.27\left(3^{\circ}\right)$ & $12.04 \pm 2.06$ \\
\hline LCL strain (\%) & Peak strain & $25.87 \pm 3.33$ & $8.59 \pm 2.21\left(5^{\circ}\right)$ & $4.92 \pm 0.96$ & $9.12 \pm 1.45\left(3^{\circ}\right)$ & $22.04 \pm 2.35$ \\
\hline
\end{tabular}

significantly affected when the medial or the lateral tibial plateau was elevated beyond three degrees: An elevated medial plateau resulted in increased MCL peak strain, but decreased LCL peak strain, whereas an elevated lateral plateau resulted in a decreased MCL peak strain and increased LCL peak strain (Figure 5A).

\subsection{Knee alignment}

\subsubsection{Coronal plane}

KAM was significantly altered beyond three degrees and one degree varus and valgus alignment at first and second peak, respectively. KAM significantly increased with increased varus alignment, whereas decreased KAM was observed with increased valgus alignment (Figure 2B, Table 1).

Medial-lateral force and pressure distribution were significantly altered beyond three degrees varus and valgus alignment: medial contact force and pressure significantly increased with increased varus alignment, while the lateral contact force and pressure decreased (Figure 3B and 4B, Table 1). Lateral contact force and pressure significantly increased with increased valgus alignment, while medial contact force and pressure decreased (Figure 3B and 4B). Results for the effect on the total knee contact force are reported in supplementary material B.

ACL peak strain was significantly increased beyond nine degrees varus and five degrees valgus. PCL peak strain was significantly increased beyond seven degrees varus and valgus, however at three degrees varus PCL peak strain was significantly decreased (Figure 5B, Table 1). MCL peak strain was significantly increased beyond one degree varus and seven degrees valgus. However, at three degrees valgus MCL peak strain was significantly decreased compared to the reference simulation. LCL peak strain was significantly increased beyond five degrees varus and three degrees valgus (Figure 5B).

\subsubsection{Transverse plane}

Altering knee alignment in the transverse plane only significantly affected KAM and the load distribution during the second peak: KAM was increased when the knee was internally rotated beyond three degrees, whereas KAM was decreased when the knee was externally rotated beyond three degrees (Figure 2C, Table 2).

Medial contact force was increased when the knee was internally rotated beyond seven degrees internal rotation, while lateral contact force was decreased when the knee was internally rotated beyond nine degrees (Figure 3C). Lateral contact force was 

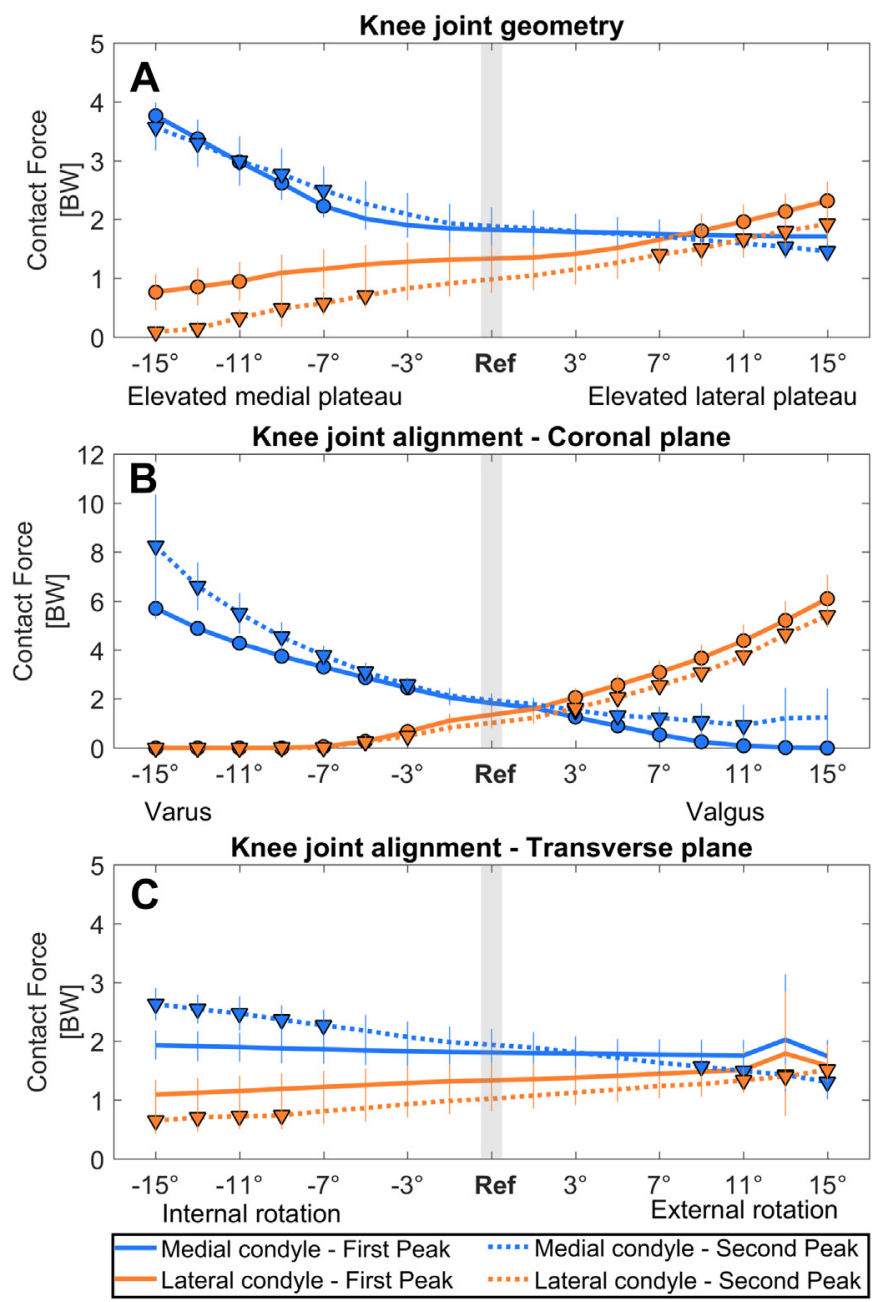

Figure 3. Effect on the contact force distribution. The effect of an altered joint geometry in A) the coronal plane, $\mathrm{C}$ ) the transverse plane and an altered joint alignment in B) the coronal plane and D) the transverse plane on the knee contact force distribution at the first peak (FP, solid line) and at the second peak (SP, dashed line). The blue line shows the effect on the medial condyle contact force, and the orange line shows the effect on the lateral condyle contact force. A significant difference compared to the contact force during the reference simulation (gray bar) is indicated by a solid dot (first peak) and a solid triangle (second peak). ( $\alpha_{B C}$ $=0.0031$ ). (For interpretation of the references to color in this figure legend, the reader is referred to the web version of this article.)

increased when the knee was externally rotated beyond $11^{\circ}$, but medial contact force was decreased when the knee was externally rotated beyond nine degrees (Figure 3C). Medial mean pressure was increased when the knee was internally rotated beyond nine degrees internal rotation. Lateral mean pressure was increased when the knee was externally rotated beyond nine degrees, but medial mean pressure was decreased when the knee was externally rotated beyond $11^{\circ}$ (Figure $4 \mathrm{C}$ ). Results for the effect on the total knee contact force are reported in supplementary material B.

ACL peak strain was significantly increased when the knee was internally rotated beyond $11^{\circ}$ and was only significantly decreased at $13^{\circ}$ external rotation. PCL peak strain was significantly increased when the knee was externally rotated beyond $13^{\circ}$ (Figure 5C). MCL peak strain was significantly increased when the knee was internally rotated beyond nine degrees, whereas LCL peak strain was not significantly affected (Figure 5C).

\section{Discussion}

The present study indicates that the medial-lateral tibiofemoral load distribution and ligament strains during walking are sensitive to both the joint geometry and alignment in the coronal plane, representative for coronal tibial slope and knee varus-valgus malalignment, whereas it is less sensitive to changes in knee alignment in the transverse plane. Since OA initiation and progression have previously been attributed to excessive mechanical loading induced by joint malalignment and geometry, these results may contribute to a better understanding of the relation between deviating knee geometry or alignment and altered knee loading and ultimately onset and progression of degenerative cartilage diseases.

Relatively small alterations in coronal plane knee alignment significantly affect the knee loading distribution. In neutral alignment, more loading is taken up by the medial condyle [34]. Coronal plane knee malalignment significantly affected the medial- 

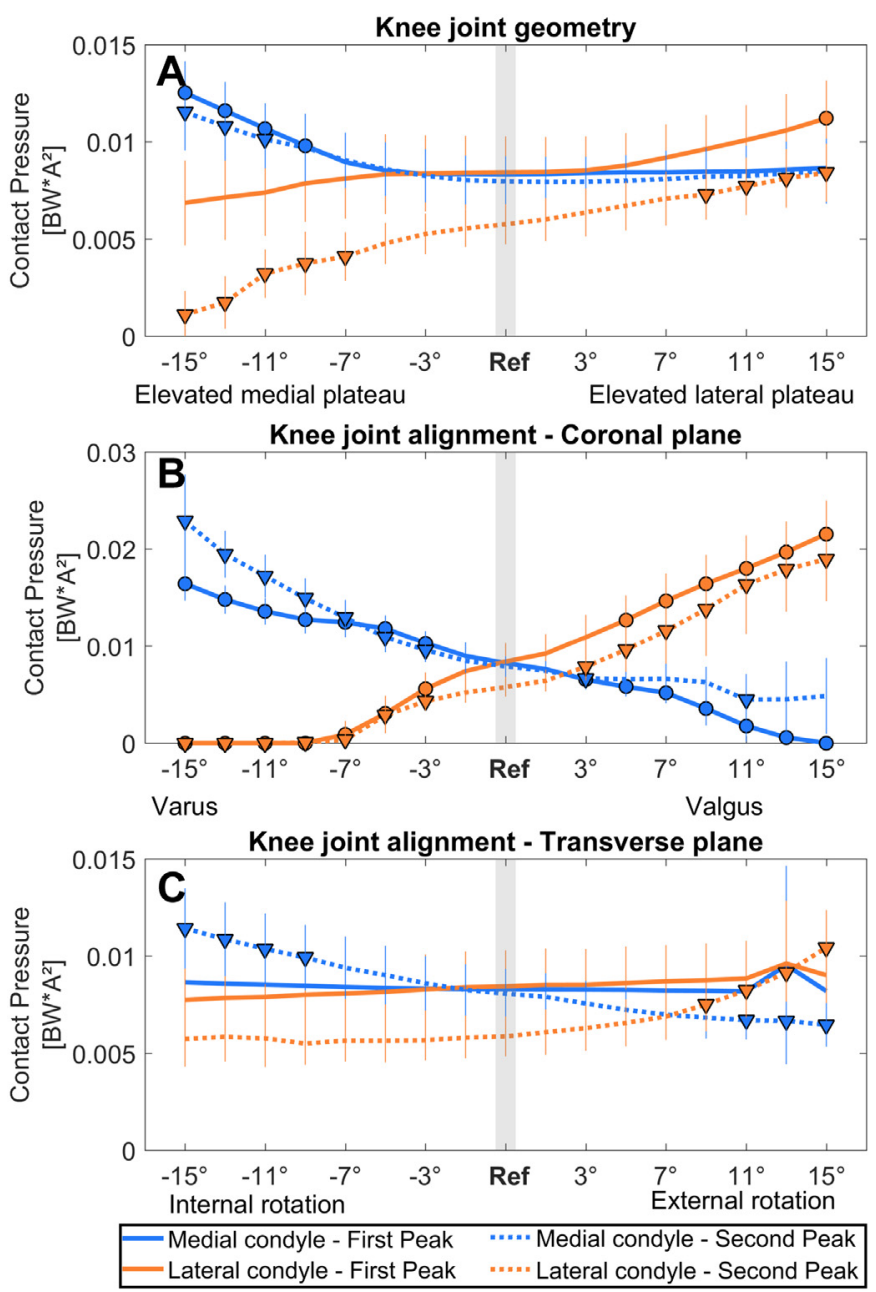

Figure 4. Effect on the contact pressure distribution. The effect of an altered joint geometry in A) the coronal plane, C) the transverse plane and an altered joint alignment in B) the coronal plane and D) the transverse plane on the knee contact pressure distribution at the first peak (FP, solid line) and at the second peak (SP, dashed line). The blue line shows the effect on the medial condyle contact pressure, and the orange line shows the effect on the lateral condyle contact pressure. A significant difference compared to pressure during the reference simulation (gray bar) is indicated by a solid dot (first peak) and a solid triangle (second peak). $\left(\alpha_{B C}=0.0031\right)$. (For interpretation of the references to color in this figure legend, the reader is referred to the web version of this article.)

lateral force distribution beyond three degrees additional varus or valgus compared to the reference position. Additional varus resulted in increased medial condyle loading, while additional valgus resulted in increased lateral condyle loading, with a simultaneous load reduction on the opposite condyle. As a measure of medial condyle loading, KAM was previously found to be directly proportional to the degree of coronal plane malalignment [11]. In support of this, our results showed a clear dependence of coronal plane knee alignment on the load distribution in terms of knee contact forces as well as KAM. This indicates that coronal plane malalignment could impose excessive stress on the articular cartilage and subchondral bone and could therefore potentially contribute to knee OA disease initiation and progression [5]. Additionally, altering coronal plane alignment resulted in increased ligament strains of all four ligaments. This suggests that increased malalignment causes increased movement in the knee that needs to be stabilized by all ligaments, causing the increased strains. Previously, knee instability was observed in patients with established knee OA [21]. As the ligaments are experiencing more strains during walking in presence of coronal plane knee malalignment, this would suggest that the chronic excessive stretching of the ligaments could contribute to additional knee instability, which could accelerate the degenerative changes in the cartilage.

Altered coronal plane joint geometry, indicative of altered coronal tibial slope, also significantly affected the medial-lateral force distribution, although to a lesser extent than the knee joint alignment. A more elevated medial tibia plateau resulted in increased medial loading and decreased lateral loading, whereas the opposite was observed with a more elevated lateral tibia plateau. The medial-lateral pressure distribution was affected in a similar way as the medial-lateral force distribution, although the pressure distribution was only significantly affected from more severe changes in coronal tibial slope. This can be explained by the fact that next to the contact force distribution the contact area was significantly increased and consequently diminishing the influence of the alterations in coronal tibial slope on the pressure distribution (Contact area changes are reported in supplementary material C). Remarkably, in the simulations of an altered joint geometry, KAM was not significantly changed, whereas the medial- 

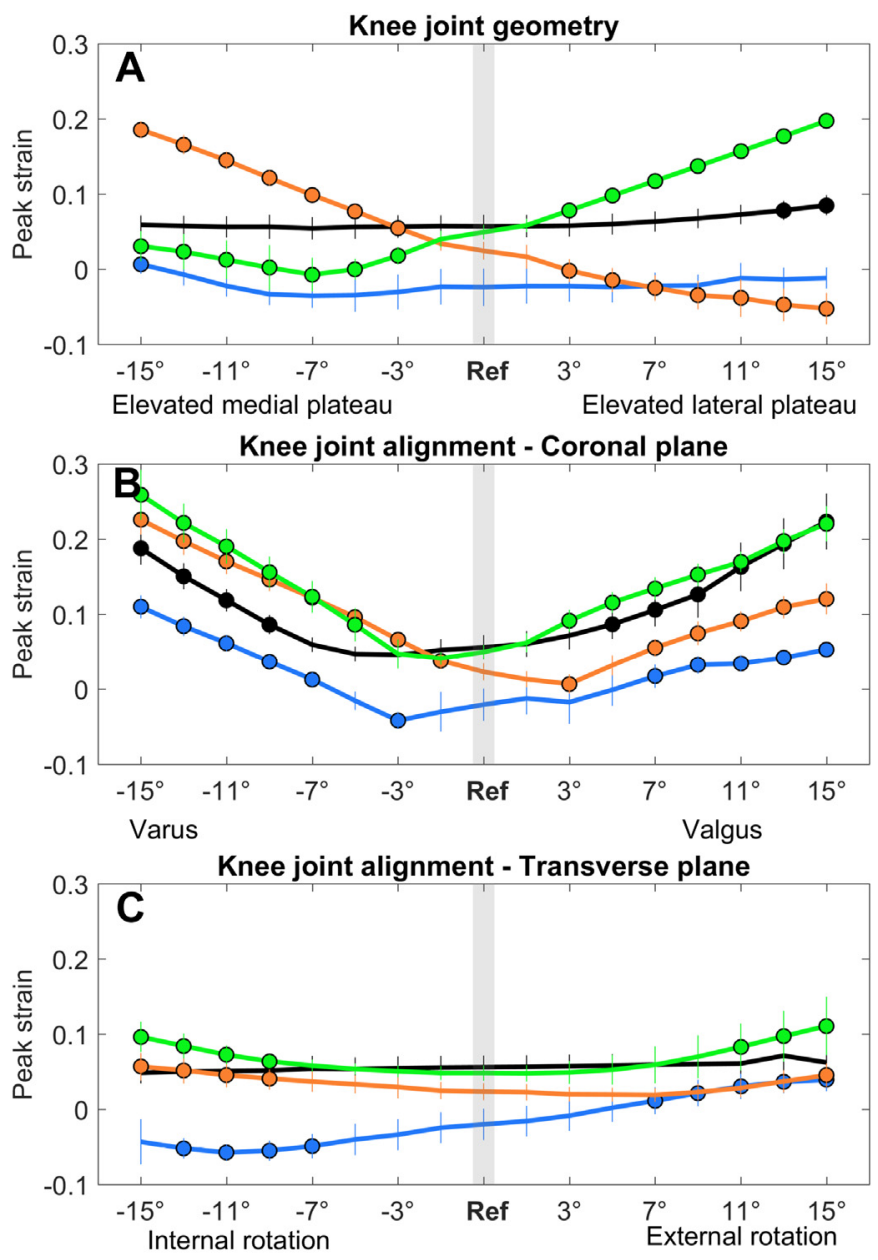

$-\mathrm{ACL} \quad-\mathrm{PCL}=\mathrm{MCL}=\mathrm{LCL}$

Figure 5. Effect on the ligament strains. The effect of an altered joint geometry in A) the coronal plane, C) the transverse plane and an altered joint alignment in B) the coronal plane and D) the transverse plane on the peak ligament strain. The black line represents the ACL, the blue line the PCL, the orange line the MCL and the green line the LCL. A significant difference compared to the ligaments strains during the reference simulation (gray bar) is indicated by a solid dot. $\left(\alpha_{B C}=0.0031\right)$. (For interpretation of the references to color in this figure legend, the reader is referred to the web version of this article.)

Table 2

Altered malalignment conditions in the transverse plane resulting in significantly altered loading conditions.

\begin{tabular}{|c|c|c|c|c|c|c|}
\hline \multirow[t]{2}{*}{ Transverse plane - alignment } & & \multirow{2}{*}{$\frac{\text { Extreme }}{\text { Internal rotation }}$} & \multirow[t]{2}{*}{ Significant from } & \multirow[t]{2}{*}{ Reference value } & \multirow[t]{2}{*}{ Significant from } & \multirow{2}{*}{$\begin{array}{l}\text { Extreme } \\
\text { External rotation }\end{array}$} \\
\hline & & & & & & \\
\hline \multirow{3}{*}{ Knee adduction moment (Nm/kg) } & First Peak & - & - & $-0.28 \pm 0.09$ & - & - \\
\hline & Second Peak & $-0.58 \pm 0.08$ & $-0.24 \pm 0.06\left(3^{\circ}\right)$ & $-0.17 \pm 0.06$ & $-0.09 \pm 0.06\left(3^{\circ}\right)$ & $0.21 \pm 0.07$ \\
\hline & First Peak & - & - & $1.81 \pm 0.26$ & - & - \\
\hline \multirow[t]{2}{*}{ Medial contact force (BW) } & Second Peak & $2.63 \pm 0.27$ & $2.27 \pm 0.27\left(7^{\circ}\right)$ & $1.94 \pm 0.27$ & $1.57 \pm 0.28\left(9^{\circ}\right)$ & $1.31 \pm 0.29$ \\
\hline & First Peak & - & - & $1.34 \pm 0.3$ & - & - \\
\hline \multirow[t]{2}{*}{ Lateral contact force (BW) } & Second Peak & $0.65 \pm 0.22$ & $0.74 \pm 0.24\left(9^{\circ}\right)$ & $1.02 \pm 0.21$ & $1.34 \pm 0.22\left(11^{\circ}\right)$ & $1.51 \pm 0.21$ \\
\hline & First Peak & - & - & $0.008 \pm 0.001$ & - & - \\
\hline \multirow[t]{2}{*}{ Medial contact pressure $\left(B W * A^{2}\right)$} & Second Peak & $0.011 \pm 0.002$ & $0.01 \pm 0.002\left(9^{\circ}\right)$ & $0.008 \pm 0.001$ & $0.007 \pm 0.001\left(11^{\circ}\right)$ & $0.006 \pm 0.001$ \\
\hline & First Peak & - & - & $0.008 \pm 0.002$ & - & - \\
\hline Lateral contact pressure $\left(\mathrm{BW} * \mathrm{~A}^{2}\right)$ & Second Peak & - & - & $0.006 \pm 0.001$ & $0.008 \pm 0.001\left(9^{\circ}\right)$ & $0.01 \pm 0.002$ \\
\hline ACL strain (\%) & Peak strain & - & - & $5.59 \pm 1.56$ & - & - \\
\hline PCL strain (\%) & Peak strain & - & $-4.91 \pm 1.62\left(7^{\circ}\right)$ & $-2.01 \pm 2.08$ & $1.12 \pm 1.79\left(7^{\circ}\right)$ & $3.92 \pm 1.53$ \\
\hline MCL strain (\%) & Peak strain & $5.67 \pm 1.83$ & $4.09 \pm 1.5\left(9^{\circ}\right)$ & $2.34 \pm 1.13$ & $4.52 \pm 1.59\left(15^{\circ}\right)$ & $4.52 \pm 1.59$ \\
\hline LCL strain (\%) & Peak strain & $9.61 \pm 2.02$ & $6.37 \pm 0.89\left(9^{\circ}\right)$ & $4.82 \pm 1.03$ & $8.29 \pm 3.11\left(11^{\circ}\right)$ & $11.08 \pm 3.88$ \\
\hline
\end{tabular}


lateral force distribution was significantly changed. This shows the benefit of calculating the contact force distribution instead of solely relying on KAM as an estimate of medial condyle loading. Therefore, medial contact force could be a more sensitive biomarker for early detection of OA, instead of using KAM. This would allow an earlier detection of OA and consequently interventions to delay OA progression could be taken earlier. Indeed, an increased risk for accelerated knee OA was already found among patients with malalignment and an increased coronal tibial slope, whereas this relation was not present in patients with a neutrally aligned knee [6]. This observation is in line with our result that the loading distribution was not significantly affected when tibial slope was altered in the range of tibial slopes previously observed in the patients with neutral knee alignment. This suggests that in knees with neutral alignment, small deviations in the coronal tibia angle may be tolerated or that the effect on the cartilage loading alone is not sufficient to contribute to degenerative changes in the cartilage.

Ligament behavior is strongly affected by the presence of altered coronal tibial slope, potentially imposing chronic stress and long-lasting stretch on the knee ligaments during daily life activities as walking. Peak strains of the collateral ligaments were significantly affected by small changes in the coronal tibia angle, which is representative for an altered joint geometry. A more elevated medial tibial plateau resulted in significantly increased MCL peak strains, whereas a more elevated lateral tibial plateau resulted in significantly increased LCL peak strains. As a consequence, this could potentially introduce coronal plane knee laxity and potentially resulting in increased knee instability, which were previously identified as risk factor for knee OA initiation and progression $[21,35]$. Hence, coronal plane knee laxity likely adds to a vicious circle, with articular cartilage degeneration contributing to joint malalignment and subsequently altered joint loading and increasing articular cartilage breakdown [21,36]. This would imply that subtle alterations in joint geometry could introduce joint malalignment later in life, which in turn would introduce altered loading and accelerate OA progression.

The effects of altered alignment in the transverse plane on the loading distribution was less pronounced compared to the alterations in the coronal plane. When the transverse plane alignment was altered, the force distribution was only significantly affected during terminal stance. From seven degrees internal rotation and onwards, medial condyle loading was increased accompanied by decreased lateral loading. Besides a small effect on the loading distribution, altered knee alignment in the transverse plane also increased the peak strains of the MCL and LCL. As in the coronal plane, this likely contributes to transverse plane laxity, which causes knee instability. Similarly to an altered transverse plane knee alignment, knee instability in the transverse plane was previously observed after ACL-injury. This is reflected in altered knee kinematics and can be interpreted as a change in transverse plane alignment [37,38]. Altered contact regions were previously observed after ACL-injury [39]. Since these cartilage regions are less adapted to the loading, degenerative changes in the cartilage could occur and might partially contribute to the increased incidence of OA after ACL-injury [23,40-42]. This suggests that transverse plane alignment might contribute to $\mathrm{OA}$ initiation by affecting knee stability (by its effect on the ligaments) and therefore contact locations instead of the loading magnitude.

The current results provide additional insight in the isolated effect of an altered joint geometry or joint malalignment on the distribution of loading over the articular cartilage, however the current results should be evaluated with respect to some limitations. Firstly, the approach used in the current study to simulate the effect of different knee alignments, did not include any patient specific compensations in the hip, knee or ankle kinematics to reduce knee loading. Therefore, our results are representative for a situation without compensatory gait mechanisms and possibly overestimate the effect of altered knee alignment on the loading distribution. Secondly, the effect of alterations in knee alignment on joint loading might be overestimated as the orientation of the ground reaction force was not changed along with the change in center of pressure location with respect to the foot. However, our assumption that trunk movement would account for any dynamic inconsistency introduced by repositioning the tibia seems feasible, as increased trunk movement was previously observed in OA patients with malalignment [11]. Thirdly, a generic musculoskeletal model, consisting of generic bone and cartilage meshes was used. Therefore, the variability in contact force distribution between subjects is only affected by individual differences in kinematics and external forces rather than by differences in cartilage thickness distribution.

The present study illustrated a clear dependence of coronal plane joint alignment on the medial-lateral force distribution. Increased varus alignment, starting from three degrees varus, was related to increased medial loading, providing further insight in the relation between varus malalignment and medial knee OA. In addition, small changes in coronal plane tibial geometries resulted directly in increased ligament strains. These findings suggest a possible influence of coronal tibial slope on ligament laxity on the long term, which could contribute to joint malalignment and consequently altered joint loading. An altered joint geometry or alignment in the coronal plane had a more pronounced effect on knee joint loading compared to alterations in the transverse plane. This may imply that deviations in the coronal plane are more important contributors to knee OA disease initiation and progression, compared to deviations in the transverse plane.

\section{CRediT authorship contribution statement}

Sam Van Rossom: Conceptualization, Data curation, Formal analysis, Investigation, Methodology, Validation, Visualization, Writing - original draft, Writing - review \& editing. Mariska Wesseling: Data curation, Formal analysis, Investigation, Methodology, Validation, Visualization, Writing - review \& editing. Colin R. Smith: Data curation, Methodology, Software, Validation, Writing - review \& editing. Darryl G. Thelen: Data curation, Funding acquisition, Methodology, Software, Validation, Writing - review \& editing. Benedicte Vanwanseele: Methodology, Project administration, Resources, Writing - review \& editing. Van Assche Dieter: Investigation, Methodology, Supervision, Writing - review \& editing. Ilse Jonkers: Conceptualization, Funding acquisition, Methodology, Project administration, Resources, Supervision, Writing - review \& editing. 


\section{Acknowledgments}

\section{Role of the funding source}

Research was supported by funding of the KU Leuven research council (OT/13/083), Whitaker International Summer Program (CS) and NIH Grant EB015410

\section{Declaration of Competing Interest}

The authors declare that no conflicts of interest exist.

\section{Appendix A. Supplementary data}

Supplementary data to this article can be found online at https://doi.org/10.1016/j.knee.2019.06.002.

\section{References}

[1] Hunter DJ, Felson DT. Osteoarthritis. BMJ 2006;332:639-42. https://doi.org/10.1136/bmj.332.7542.639.

[2] Baker-LePain JC, Lane NE. Role of bone architecture and anatomy in osteoarthritis. Bone 2012;51:197-203. https://doi.org/10.1016/j.bone.2012.01.008.

[3] Brouwer GM, Van Tol AW, Bergink AP, Belo JN, Bernsen RMD, Reijman M, et al. Association between valgus and varus alignment and the development and progression of radiographic osteoarthritis of the knee. Arthritis Rheum 2007;56:1204-11. https://doi.org/10.1002/art.22515.

[4] Hunter D, Nevitt M, Lynch J, Kraus VB, Katz JN, Collins JE, et al. Longitudinal validation of periarticular bone area and 3D shape as biomarkers for knee OA progression? Data from the FNIH OA biomarkers consortium. Ann Rheum Dis 2015. https://doi.org/10.1136/annrheumdis-2015-207602 annrheumdis-2015-207602.

[5] Andriacchi TP, Favre J. The nature of in vivo mechanical signals that influence cartilage health and progression to knee osteoarthritis. Curr Rheumatol Rep 2014; 16:463-70.

[6] Driban JB, Stout AC, Duryea J, Lo GH, Harvey WF, Price LL, et al. Coronal tibial slope is associated with accelerated knee osteoarthritis: data from the osteoarthritis initiative. BMC Musculoskelet Disord 2016;17:1-8. https://doi.org/10.1186/s12891-016-1158-9.

[7] Haverkamp DJ, Schiphof D, Bierma-Zeinstra SM, Weinans H, Waarsing JH. Variation in joint shape of osteoarthritic knees. Arthritis Rheum 2011;63:3401-7. https://doi.org/10.1002/art.30575.

[8] Moisio K, Chang A, Eckstein F, Chmiel JS, Wirth W, Almagor O, et al. Varus-valgus alignment reduced risk of subsequent cartilage loss in the less loaded compartment. Arthritis Rheum 2011;63:1002-9. https://doi.org/10.1002/art.30216.

[9] Hayashi D, Englund M, Roemer FW, Niu J, Sharma L, Felson DT, et al. Knee malalignment is associated with an increased risk for incident and enlarging bone marrow lesions in the more loaded compartments: the MOST study. Osteoarthr Cartil 2012;20:1227-33. https://doi.org/10.1016/j.joca.2012.07.020.

[10] Moyer R, Wirth W, Duryea J, Eckstein F. Anatomical alignment, but not goniometry, predicts femorotibial cartilage loss as well as mechanical alignment: data from the osteoarthritis initiative. Osteoarthr Cartil 2016;24:254-61. https://doi.org/10.1016/j.joca.2015.08.016.

[11] Turcot K, Armand S, Lübbeke A, Fritschy D, Hoffmeyer P, Suvà D. Does knee alignment influence gait in patients with severe knee osteoarthritis? Clin Biomech 2013;28:34-9. https://doi.org/10.1016/j.clinbiomech.2012.09.004.

[12] van Egmond N, Stolwijk N, van Heerwaarden R, van Kampen A, Keijsers NLW. Gait analysis before and after corrective osteotomy in patients with knee osteoarthritis and a valgus deformity. Knee Surgery, Sport Traumatol Arthrosc 2016:1-10. https://doi.org/10.1007/s00167-016-4045-x.

[13] Weidenhielm L, Svensson OK, Broström LÅ, Rudberg U. Change in adduction moment about the knee after high tibial osteotomy and prosthetic replacement in osteoarthrosis of the knee. Clin Biomech 1992;7:91-6. https://doi.org/10.1016/0268-0033(92)90021-U.

[14] Sharma L, Song J, Felson DT, Cahue S, Shamiyeh E, Dunlop DD. The role of knee alignment in disease progression and functional decline in knee osteoarthritis. JAMA 2001;286:188-95. https://doi.org/10.1001/jama.286.2.188.

[15] Cicuttini F, Wluka A, Hankin J, Wang Y. Longitudinal study of the relationship between knee angle and tibiofemoral cartilage volume in subjects with knee osteoarthritis. Rheumatology 2004;43:321-4. https://doi.org/10.1093/rheumatology/keh017.

[16] Huizinga MR, Gorter J, Demmer A, Bierma-Zeinstra SMA, Brouwer RW. Progression of medial compartmental osteoarthritis 2-8 years after lateral closing-wedge high tibial osteotomy. Sport Traumatol Arthrosc: Knee Surgery; 2016. https://doi.org/10.1007/s00167-016-4232-9.

[17] Tanamas S, Hanna FS, Cicuttini FM, Wluka AE, Berry P, Urquhart DM. Does knee malalignment increase the risk of development and progression of knee osteoarthritis? A systematic review. Arthritis Care Res 2009;61:459-67. https://doi.org/10.1002/art.24336.

[18] Miyazaki T, Wada M, Kawahara H, Sato M, Baba H, Shimada S. Dynamic load at baseline can predict radiographic disease progression in medial compartment knee osteoarthritis. Ann Rheum Dis 2002;61:617-22.

[19] Krackow KA, Mandeville DS, Rachala SR, Bayers-Thering M, Osternig LR. Torsion deformity and joint loading for medial knee osteoarthritis. Gait Posture 2011;33: 625-9. https://doi.org/10.1016/j.gaitpost.2011.02.006.

[20] Felson DT, Lawrence RC, Dieppe PA, Hirsch R, Helmick CG, Jordan JM, et al. Osteoarthritis: new insights - part 1: the disease and its risk factors. Ann Intern Med 2000;133:635-46.

[21] Lewek MD, Rudolph KS, Snyder-Mackler L. Control of frontal plane knee laxity during gait in patients with medial compartment knee osteoarthritis. Osteoarthr Cartil 2004;12:745-51.

[22] Sharma L, Lou C, Felson DT, Dunlop DD, Kirwan-Mellis G, Hayes KW, et al. Laxity in healthy and osteoarthritic knees. Arthritis Rheum 1999;42:861-70. https://doi. org/10.1002/1529-0131(199905)42:5<861::AID-ANR4>3.0.CO;2-N.

[23] Chaudhari AMW, Briant PL, Bevill SL, Koo S, Andriacchi TP. Knee kinematics, cartilage morphology, and osteoarthritis after ACL injury. Med Sci Sports Exerc 2008; 40:215-22.

[24] Agneskirchner JD, Hurschler C, Wrann CD, Lobenhoffer P. The effects of valgus medial opening wedge high tibial osteotomy on articular cartilage pressure of the knee: a biomechanical study. Arthrosc - J Arthrosc Relat Surg 2007;23:852-61. https://doi.org/10.1016/j.arthro.2007.05.018.

[25] Riegger-Krugh C, Gerhart TN, Powers WR, Hayes WC. Tibiofemoral contact pressures in degenerative joint disease. Clin Orthop Relat Res 1998;348:233-45. https://doi.org/10.1097/00003086-199803000-00035.

[26] Thompson JA, Hast MW, Granger JF, Piazza SJ, Siston RA. Biomechanical effects of total knee arthroplasty component malrotation: a computational simulation. J Orthop Res 2011;29:969-75. https://doi.org/10.1002/jor.21344.

[27] Lenhart RL, Kaiser J, Smith CR, Thelen DG. Prediction and validation of load-dependent behavior of the tibiofemoral and patellofemoral joints during movement. Ann Biomed Eng 2015;43:2675-85.

[28] Smith RC, Choi KW, Negrut D, Thelen DG. Efficient computation of cartilage contact pressures within dynamic simulations of movement. Comput Methods Biomech Biomed Eng Imaging Vis 2016;0:1-8.

[29] Hudelmaier M, Glaser C, Englmeier K-H, Reiser M, Putz R, Eckstein F. Correlation of knee-joint cartilage morphology with muscle cross-sectional areas vs. anthropometric variables. Anat Rec Part A 2003;270:175-84. 
[30] Eckstein F, Reiser M, Englmeier KH, Putz R. In vivo morphometry and functional analysis of human articular cartilage with quantitative magnetic resonance imaging-from image to data, from data to theory. Anat Embryol (Berl) 2001;203:147-73.

[31] Li G, Lopez O, Rubash H. Variability of a three-dimensional finite element model constructed using magnetic resonance images of a knee for joint contact stress analysis. J Biomech Eng 2001;123:341-6.

[32] Adouni M, Shirazi-Adl A. Partitioning of knee joint internal forces in gait is dictated by the knee adduction angle and not by the knee adduction moment. J Biomech 2014:47:1696-703.

[33] Wesseling M, De Groote F, Meyer C, Corten K, Simon JP, Desloovere K, et al. Gait alterations to effectively reduce hip contact forces. J Orthop Res 2015;33: 1094-102. https://doi.org/10.1002/jor.22852.

[34] Schipplein OD, Andriacchi TP. Interaction between active and passive knee stabilizers during level walking. J Orthop Res 1991;9:113-9. https://doi.org/10.1002/ jor.1100090114.

[35] Bytyqi D, Shabani B, Lustig S, Cheze L, Gjurgjeala NK, Neyret P. Gait knee kinematic alterations in medial osteoarthritis: three dimensional assessment. Int Orthop 2014;38:1191-8. https://doi.org/10.1007/s00264-014-2312-3.

[36] Lewek MD, Ramsey DK, Snyder-mackler L, Rudolph KS. Knee stabilization in patients with medial compartment knee osteoarthritis. Arthritis Rheum 2005;52: 2845-53. https://doi.org/10.1002/art.21237.

[37] Yamaguchi S, Gamada K, Sasho T, Kato H, Sonoda M, Banks Sa. In vivo kinematics of anterior cruciate ligament deficient knees during pivot and squat activities. Clin Biomech (Bristol, Avon) 2009;24:71-6. https://doi.org/10.1016/j.clinbiomech.2008.08.007.

[38] Georgoulis AD, Papadonikolakis A, Papageorgiou CD, Mitsou A, Stergiou N. Three-dimensional tibiofemoral kinematics of the anterior cruciate ligament-deficient and reconstructed knee during walking. Am J Sports Med 2003;31:75-9.

[39] Van De Velde SK, Bingham JT, Hosseini A, Kozanek M, DeFrate LE, Gill T], et al. Increased tibiofemoral cartilage contact deformation in patients with anterior cruciate ligament deficiency. Arthritis Rheum 2009;60:3693-702. https://doi.org/10.1002/art.24965.

[40] Lohmander LS, Ostenberg a, Englund M, Roos H. High prevalence of knee osteoarthritis, pain, and functional limitations in female soccer players twelve years after anterior cruciate ligament injury. Arthritis Rheum 2004:50:3145-52. https://doi.org/10.1002/art.20589.

[41] von Porat a, Roos EM, Roos H. High prevalence of osteoarthritis 14 years after an anterior cruciate ligament tear in male soccer players: a study of radiographic and patient relevant outcomes. Ann Rheum Dis 2004;63:269-73. https://doi.org/10.1136/ard.2003.008136.

[42] Van Rossom S, Wesseling M, Assche D Van, Jonkers I. Topographical Variation of Human Femoral Articular Cartilage Thickness , T1rho and T2 Relaxation Times Is Related to Local Loading during Walking. Cartilage 2018. https://doi.org/10.1177/1947603517752057. 